\title{
EL POZOL, UNA BEBIDA ANCESTRAL MEXICANA COMO LEGADO CULTURAL INMATERIAL
}

\section{POZOL, AN ANCESTRAL MEXICAN DRINK AS AN INTANGIBLE CULTURAL LEGACY}

\author{
Olga Yeri González López*, Lisbeth Jacinto Castillo** \\ Marina Pérez Cano***
}

\begin{abstract}
*Maestra en Administración. Profesora-Investigadora. División Académica de Ciencias Económico Administrativas. Universidad Juárez Autónoma de Tabasco.

**Doctora en Educación. Profesora-Investigadora. División Académica de Ciencias Económico Administrativas. Universidad Juárez Autónoma de Tabasco. Email: ljacsc@hotmail.com

${ }^{* * *}$ Maestra en Administración. Profesora-Investigadora. División Académica de Ciencias Económico Administrativas. Universidad Juárez Autónoma de Tabasco. Email: marina.perez@ujat.mx
\end{abstract}

Dirección para recibir correspondencia: olga_yeri@hotmail.com 
EL POZOL, UNA BEBIDA ANCESTRAL MEXICANA COMO LEGADO CULTURAL INMATERIAL

\section{RESUMEN}

OBJETIVO: Describir la elaboración del pozol como un bien inmaterial gastronómico, colocando énfasis en la transmisión de las prácticas culturales como elemento que permite la vigencia de la cocina ancestral tabasqueña.

MATERIAL Y MÉTODO: El estudio se refiere a la elaboración artesanal del pozol, donde influyen aspectos como el tipo de materia prima, tiempo de cocción, proceso de tostado del cacao, tipo de maíz entre otros. Se utilizó como técnica, la entrevista libre estructurada a partir un cuestionamiento detonador para conocer la manera en que se transmite el conocimiento de su elaboración y la relación con la familia. El tipo de muestreo fue no probabilístico por conveniencia, porque se entrevistó a personas oriundas de tres municipios tabasqueños representativos de la región de los Ríos, la Sierra y Centro, para conocer si en estas regiones prevalece la trasmisión de las tradiciones culturales con respecto a la elaboración esta bebida.

RESULTADOS: La población entrevistada coincidió en el proceso general de elaboración del pozol. Las combinaciones del maíz con cacao, canela, cacahuate, pataste y camote resultan típicas en la región, contribuyendo a la nutrición de las familias por su contenido calórico de carbohidratos y grasas. La comercialización de esta bebida se hace en microempresas familiares en mercados, pequeños locales comerciales y en las casas, caracterizándose por ser rentable al proveer ingresos para el distribuidor sobre el 50\% de la inversión.

CONCLUSIONES: El pozol es una bebida ancestral, un legado cultural de los mayas-chontales y una oportunidad de negocios para las familias. Su valor nutricional, su representatividad como la bebida tradicional del Estado de Tabasco y su flexibilidad de combinación con diversos frutos y semillas lo hacen altamente comercializable como agua fresca y masa-base para elaboración de la bebida, creando ingreso y permitiendo el desarrollo social y comercial de la región.

PALABRAS CLAVE: Bien inmaterial. Pozol. Microempresas familiares. Cultura. 
EL POZOL, UNA BEBIDA ANCESTRAL MEXICANA COMO LEGADO CULTURAL INMATERIAL

\section{ABSTRACT}

OBJECTIVE: To describe the elaboration of pozol as an intangible gastronomic asset, placing emphasis on the transmission of cultural practices as an element that allows the validity of the ancestral cuisine of Tabasco.

MATERIAL AND METHOD: The study refers to the traditional production of pozol, where aspects such as the type of raw material, time of cooking, roasting process of cocoa, and the type of corn among others. The structured free interview was used as a technique, starting with a questioning trigger, to know the way in which the knowledge of its elaboration and the relationship with the family is transmitted. The type of sampling was intentionally not probabilistic, since people from three municipalities of Tabasco, representative of the Rios and the Sierra regions as well as Centro were interviewed, in order to know if in these regions the transmission of cultural traditions as the elaboration of this drink prevails.

RESULTS: The population interviewed agreed on the general process of the elaboration of pozol. The combinations of corn with either cocoa, cinnamon, peanuts, chayote or sweet potatoes are typical in the region, contributing to the nutrition of families by their caloric content of carbohydrates and fats. The commercialization of this drink is carried out by family micro enterprises in markets, small business premises and in the houses, being characterized as being profitable when providing income for the distributor over $50 \%$ of the investment.

CONCLUSIONS: Pozol is an ancestral drink, a cultural legacy of the Mayas-Chontales and a business opportunity for families. Its nutritional value, its representativeness as the traditional drink of the State of Tabasco and its flexibility for its combination with various fruits and seeds makes it highly marketable as a refreshing drink or as a mass-base for the drink preparation, thus creating income and allowing social and commercial development in the region.

KEYWORDS: Intangible assets. Pozol. Family microenterprises. Culture. 


\section{EL POZOL, UNA BEBIDA ANCESTRAL MEXICANA COMO LEGADO CULTURAL INMATERIAL}

\section{INTRODUCCIÓN}

La palabra cacao proviene de la azteca "cacahualt". Según la leyenda se considera el árbol más bello del paraíso azteca, al cual se le atribuían diversas virtudes como: calmar el hambre y la sed, proporcionar la sabiduría universal y curar enfermedades. El cacao pertenece al grupo de Theobroma que en griego significa "Alimento de los Dioses" (Trujillo, 2010).

El cacao es una aportación que México hizo al mundo; sin embargo, se ha perdido su importancia en cuanto a producción y uso del mismo. En la época prehispánica para los olmecas y los mayas era la bebida predilecta de la realeza, sacerdotes y guerreros. También era conocido como oro negro (OS, 2011).

Como herencia cultural en la gastronomía, el intenso sabor, la versatilidad y diversidad de aplicaciones en la repostería, la elaboración gourmet de salsas y ensaladas, y su tradición en las bebidas calientes y frías, hacen del cacao un elemento culinario ancestral simbólicamente artístico.

Dentro de las bebidas donde se utiliza el cacao, se ubica el Pozol del Sureste Mexicano (México Desconocido, 2017) compuesto por cacao tostado y molido, masa de maíz, agua, azúcar, canela y vainilla, y la manera de tomarlo es frío. Su origen es documentado en diversos escritos de la época colonial, los españoles que anduvieron por estas tierras dieron cuenta del pozol como bebida agria de los indios que los hacía resistentes al calor (Román, 2012), identificando a los mayas-chontales de Tabasco como los creadores de esta bebida, misma que encontraron con algunas variantes en los pueblos mesoamericanos que van desde lo que hoy es Oaxaca hasta Centroamérica. Hoy en día, las pozolerías son populares en Tabasco, Chiapas y sur de Veracruz.

\section{MARCO TEÓRICO}

El estudio de la cultura conlleva dos tipos de elementos, el primero es de carácter objetivo, referido a todo aquel bien material que es creado, producido o anhelado que tiene significado particular y a partir de ello su valor para un grupo humano determinado. Un ejemplo asociado a la elaboración de bebidas a bases de maíz y cacao es la jícara, cuya presentación puede ser austera o labrada con temas históricos, sociales y religiosos. El segundo es el subjetivo y se encuentra en el interior de los sujetos, que a veces pasan o son desapercibidos por ellos mismos. Son elementos igual o más fuertes que los elementos objetivos. Ellos se reflejan en 
EL POZOL, UNA BEBIDA ANCESTRAL MEXICANA COMO LEGADO CULTURAL INMATERIAL

sus palabras y acciones; es decir, se objetivan en la conducta de los sujetos (Roman, D., 2007). Un claro ejemplo de ello es la gastronomía y particularmente el pozol que pertenece a las bebidas.

Como señala la Declaración de México sobre las Políticas Culturales "El patrimonio cultural inmaterial, comprende tradiciones o expresiones vivas heredadas de nuestros antepasados y trasmitidas a nuestros descendientes, es un factor importante del mantenimiento de la diversidad cultural frente a la creciente globalización" (Proyectos de Derechos Culturales: Cultura y Desarrollo, 1982). En el caso de Tabasco, uno de estos elementos es la elaboración del pozol, bebida tradicional que se realiza con dos componentes elementales originarios de estas tierras; cacao y maíz.

La comprensión del patrimonio cultural inmaterial de diferentes comunidades contribuye al diálogo entre culturas y promueve el respeto hacia diferentes modos de vida. No estriba únicamente en la manifestación cultural, sino en el acervo de conocimientos y técnicas que se transmiten de generación en generación.

Como se señala en la conferencia Mundial de políticas culturales (1982, p 3), "Todo pueblo tiene el derecho y el deber de defender y preservar su patrimonio cultural, ya que las sociedades se reconocen a sí mismas a través de los valores". Un patrimonio cultural intangible que debe preservarse es la gastronomía y en Tabasco particularmente sus bebidas que tienen al cacao como componente básico.

\section{METODOLOGÍA}

Se trata de una mirada introspectiva a los procesos culturales de tipo gastronómico del pueblo de Tabasco, centrando la mirada en la bebida ancestral milenaria a base de maíz y cacao, típicamente conocida como pozol. Se revisó bibliografía, se entrevistó a quienes elaboran, expenden y sirven el producto, ya sea en el hogar, una reunión familiar, un evento con visitantes de otras latitudes que, en la mayoría de los casos sucumben ante el éxtasis de sabores que detonan en el paladar ante la combinación del pozol y los dulces tradicionales, que suelen acompañarlo en su degustación; es decir, se realiza una descripción con un enfoque cualitativo, cuyo objetivo principal es describir la elaboración del pozol como un bien inmaterial gastronómico, colocando énfasis en la transmisión de las prácticas culturales como elemento que permite la vigencia de la cocina ancestral tabasqueña. 
EL POZOL, UNA BEBIDA ANCESTRAL MEXICANA COMO LEGADO CULTURAL INMATERIAL

Esta investigación es de tipo descriptivo, partiendo de la premisa de que toda cultura o sistema social tiene un modo único de entender situaciones y eventos, con la finalidad de conocer cómo se da la trasmisión de valores en cada familia en la elaboración y consumo del pozol en la experiencia de cada sujeto de estudio. Trata de indagar datos cualitativos que no pueden darse de manera numérica porque se refiere a la elaboración de la bebida, pues no existe información que pudiera representarse estadísticamente por ser elaborado de manera artesanal donde influyen aspectos como el tipo de materia prima, tiempo de cocción, proceso de tostado del cacao, tipo de maíz, entre otros.

Para obtener los resultados de esta investigación, se utilizó como técnica la entrevista libre la cual consiste en una conversación guiada con la finalidad de comprender mejor la realidad y proporcionar un marco dentro del cual los entrevistados puedan expresar su propio modo de sentir con sus propias palabras lo que permitió la recolección de datos de manera generalizada dirigida a sujetos elegidos que se relacionen con el objeto de estudio. Las entrevistas se estructuraron a partir un cuestionamiento detonador para conocer la manera en que se trasmite el conocimiento de la elaboración del pozol y la relación con la familia.

Para este estudio se seleccionó el muestreo no probabilístico por conveniencia, el cual se caracteriza por obtener muestras representativas mediante la inclusión de grupos típicos, seleccionando directa e intencionalmente la población. La muestra seleccionada se obtuvo a través de personas que laboran en el mismo centro de trabajo y que son oriundas de los municipios de Centro, Macuspana y Tenosique para conocer si en estas regiones prevalece la trasmisión de las tradiciones culturales con respecto a la elaboración del pozol.

\section{RESULTADOS}

De acuerdo a las mujeres entrevistadas, a las cuales se les realizó una pregunta detonadora, que permitiría indagar si conocían cual era el proceso de elaboración del pozol a partir de su experiencia familiar y la conducta observada de sus vivencias, se obtuvieron los siguientes resultados:

La población entrevistada coincidió en el proceso general de elaboración del pozol. Se parte de la materia prima básica: maíz desgranado, cal, cacao y agua. En primer término, se procede a la cocción del maíz con agua por un periodo una hora aproximadamente, se le agrega una cucharada de cal y se deja a fuego medio hasta que el grano de maíz se revienta y se desprende su cáscara. 
EL POZOL, UNA BEBIDA ANCESTRAL MEXICANA COMO LEGADO CULTURAL INMATERIAL

Al retirar del fuego, debe enfriarse y lavarse para quitar la cal y la cascarilla del maíz. Una vez limpio se procede a su molienda, que puede ser en molino manual o eléctrico, el cual debe ser estar ajustado para que al triturar los granos se transformen en una masa blanca. Es en esta etapa cuando se agregan los granos de cacao tostado y descascarillado y se mezcla por un proceso de amasado para fusionar todos los ingredientes.

El proceso de tostado del cacao es a fuego lento y movimientos circulares en una superficie plana, para evitar que se queme. Es vital que se esté monitoreando durante el proceso de tostado, el olor del cacao, ya que de quedar crudo, el pozol tendrá un tono claro y su proceso de fermentación será acelerado por el cacao mismo. Esto no es bueno, ya que se espera que la bebida permanezca fresca sin "agriarse" por varias horas. Sin embargo, si el cacao ha sido tostado correctamente, al mezclarse con la masa, le da una coloración café oscura, un sabor más suave y un aroma más intenso; y lo más importante, permanecerá fresco por más horas, a pesar del intenso calor.

Cabe destacar, que en el gusto de los tabasqueños está el pozol blanco, el pozol con cacao, con cacao y canela, con cacahuate, con pataste, y cualquier otro ingrediente que a su gusto podría mejorar el sabor del maíz cocido y molido. La creatividad que se imprima en el proceso de elaboración de esta bebida depende de los ingredientes que se dispongan y la habilidad de la cocinera.

Otro elemento importante a considerar de esta bebida, es su aporte nutricional a la dieta del tabasqueño, ya que no solo es deliciosa para las familias que la consideran, sino que muchas veces se constituye en el alimento principal del jornalero que trabaja en el campo. Aún se estila en algunas rancherías que el trabajador lleve en su itacate una bola de pozol y que al intermedio o término de su jornada, ésta sea preparada como bebida y alimento por la sensación de saciedad que produce, al incluir carbohidratos y grasas en una sola presentación.

En las familias entrevistadas, además de ser una bebida y formar parte de la cocina ancestral, el producto denominado "pozol" blanco o con cacao, constituye una fuente de ingreso, pues lo comercializan en su comunidad debido a que representa para esas generaciones el alimento básico, aunado a la venta de tortillas. En una economía de autoconsumo, que era común que toda la familia se involucrara en el proceso de siembra, cosecha y desgranado del maíz, así como secado y tostado del cacao. Hoy en día, con los procesos de distribución más especializados las productoras de pozol, compran el maíz ya desgranado y el cacao seco. 
EL POZOL, UNA BEBIDA ANCESTRAL MEXICANA COMO LEGADO CULTURAL INMATERIAL

La tradición de elaboración del pozol se ha realizado durante generaciones por parte de padre y madre trasmitiéndose este valor cultural de familia en familia, debido que era el alimento que el campesino llevaba al campo para nutrirse en su descanso después de una jornada de trabajo y este era batido a la mano en jícara. Se menciona por estas familias, que la mujer que no sabía hacerlo era motivo de discusiones y maltrato y catalogada como floja y reprochaban a la madre de no enseñarla a trabajar.

La generación actual ha perdido esa tradición cultural en la elaboración del "pozol" como un alimento básico, y ahora solo representa un antojo de vez en cuando, y ya no constituye una exigencia para la mujer saberlo elaborar. El pozol ha pasado de ser un producto comercializable que las familias pueden adquirir en mercados, micro-negocios familiares que se han convertido en rentable, cuyos ingresos para el distribuidor es sobre el 50\% de la inversión.

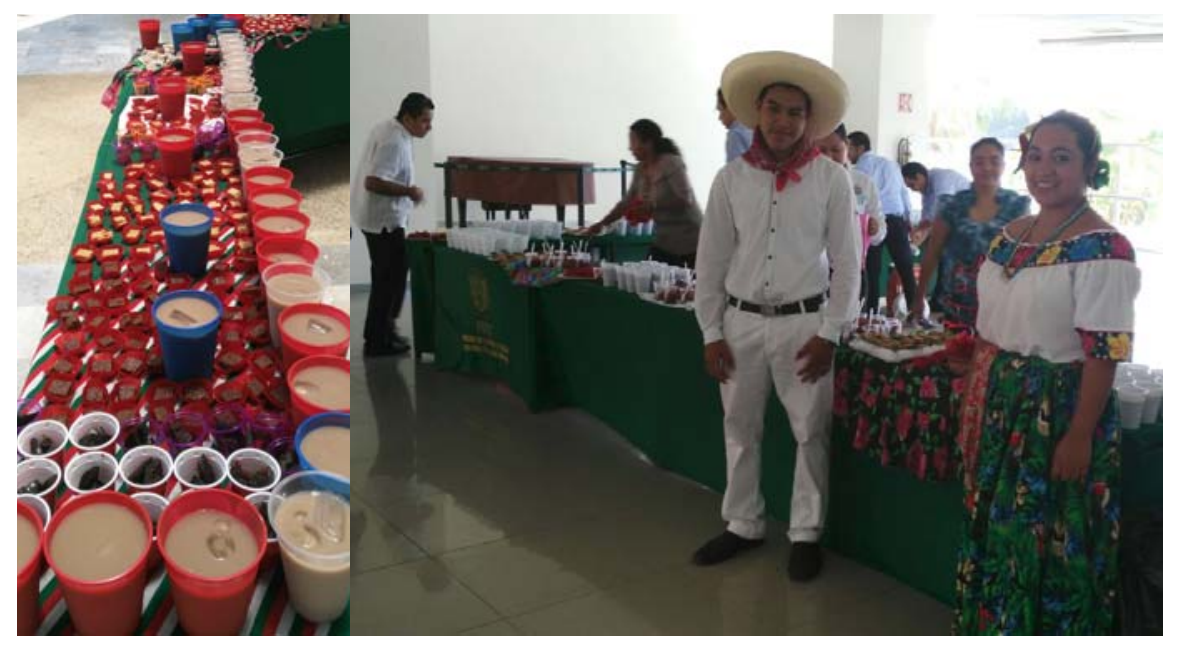

\section{CONCLUSIONES}

El carácter refrescante y nutricional del pozol, no solo apoya su conservación como parte de la alimentación del pueblo de Tabasco, sino que además lo identifica y lo distingue. La familia tabasqueña es consumidora de una bebida ancestral, cuyo proceso de elaboración actual es a partir de un subproducto de las microempresas familiares que pugnan por no perder la tradición.

Este bien inmaterial comercializable, es un alimento y crea ingreso para las familias, permitiendo el desarrollo social y comercial de la región. En el caso del municipio de Tenosique, es posible no solo beber el pozol, sino degustarlo como postre en una novedosa nieve de pozol que se puede comprar en el parque central con los distribuidores locales. 
EL POZOL, UNA BEBIDA ANCESTRAL MEXICANA COMO LEGADO CULTURAL INMATERIAL

Existe una ruptura generacional en la elaboración del pozol; sin embargo, es una fuente de ingreso y en cada región se combina de formas diversas, se le agregan nuevos ingredientes, se presenta con nuevos maridajes, pero sin perder su esencia, nos sigue recordando que según el Popol-Vuh, somos hijos de los hombres del maíz, y que el cacao representa la divinidad que los dioses un día compartieron con la humanidad.

\section{REFERENCIAS BIBLIOGRÁFICAS}

México Desconocido. (2017). México Desconocido. El pozol bebida ancestral del sureste. Recuperado de https://www.mexicodesconocido.com.mx/el-pozol-bebida-ancestral-delsureste-mexicano-tabasco.html

OS, F. (14 de 04 de 2011). blog CACAO. UPAEP, Ed. Recuperado de http://cacaomexico.blogspot.mx/

Proyectos de Derechos Culturales: Cultura y Desarrollo. (1982). Declaración de México sobre las Políticas Culturales. Conferencia Mundial sobre la Políticas Culturales, p. 6 México: Info@culturalrights.net. Recuperado

de http://www.culturalrights.net/es/documentos.php?c=18\&p=190

Roman, D. (02 de Julio de 2007). Reconceptualización de cultura popular através de son Jarocho y el Fandango. P. 90. Recuperado de UJAT, Ed., \& Tesis, Recopilador Villahermosa, Tabasco, México: Talleres Gráficos.

Román, V. (2012). Sabores de México y el mundo. Obtenido de El Pozol: Bebida Ancestral. Recuperado de http://lossaboresdemexico.com/el-pozol-bebida-ancestral/

Trujillo, J. (20 de 05 de 2010). Directo al paladar el sabor de la vida. Recuperado de https://www.directoalpaladar.com/cultura-gastronomica/breve-historia-del-cacao-y-delchocolate. 